\title{
Developing the first UK integrated respiratory registrar role in an inner city integrated care organisation
}

\author{
Authors: Melissa Heightman, Louise Restrick and Myra Stern
}

\begin{abstract}
Aims
In line with the principles of 'better communication and cooperation between community services and hospitals; between different teams working in the same buildings and between the NHS, local government and voluntary organisations', the British Thoracic society (BTS) has promoted the establishment of integrated consultant posts to optimise management of respiratory long-term conditions. Two integrated consultant posts were developed at an inner city hospital to provide close support of community respiratory teams and work directly with GPs, community services and acute medicine within the hospital. They identified a need to develop career pathways for future respiratory clinicians interested in providing integrated care and therefore aimed to pilot a novel integrated registrar position, to construct an optimised integrated job plan and ensure adequate mentorship.
\end{abstract}

\section{Methods}

A bid was put to the London Training Committee for the new integrated registrar position, which was approved for a 6-month pilot and then extended to 12 months, commencing April 2013. A job plan was designed to meet training requirements in respiratory and general internal medicine and to incorporate integrated working practice. Clinical and educational supervision was by one of the integrated consultants.

\section{Results}

A final-year flexible trainee was appointed to a supernumerary position. The 'integrated' training included: supervision of community respiratory multidisciplinary team meetings (virtual ward rounds); home visits for complex patients; delivering practice-based GP respiratory education and case-based specialist advice (virtual clinics); and training practice, district and care home nurses in respiratory clinical skills. Service development training included establishment of a pathway for community management of appropriate patients with acute exacerbations of COPD, and introducing an electronic database app for smart devices to allow better documentation of patient visits and MDT discussions and to improve information sharing between primary and secondary care. Training requirements for award of CCT were met and learning was shared with other trainees at training days, where $70 \%$ of registrars polled expressed interest in taking up a similar post. The role was documented by the RCP Future Hospital project, the King's Fund and the BTS Integrated Working Group. A second integrated registrar was subsequently appointed.

\section{Conclusions}

The registrar role provided unique training opportunities in integrated respiratory medicine, was logistically successful and was reported to have improved practice for future consultantlevel working. Ensuring future career development in integrated respiratory medicine and building clinical leadership for longterm conditions and multimorbidity will depend on extending and embedding similar training programmes.

\section{Conflict of interest statement}

No conflicts declared. 